\title{
Metal enrichment of the intra-cluster medium over a Hubble time for merging and relaxed galaxy clusters
}

\author{
W. Kapferer ${ }^{1}$, T. Kronberger ${ }^{1}$, J. Weratschnig ${ }^{1}$, S. Schindler ${ }^{1}$, W. Domainko ${ }^{2}$, \\ E. van Kampen ${ }^{1}$, S. Kimeswenger ${ }^{1}$, M. Mair ${ }^{1}$, and M. Ruffert ${ }^{3}$
}

\author{
1 Institut für Astro- und Teilchenphysik, Universität Innsbruck, Technikerstr. 25, 6020 Innsbruck, Austria \\ e-mail: Wolfgang.E.Kapferer@uibk.ac.at \\ 2 Max-Planck-Institut für Kernphysik, Saupfercheckweg 1, 69117 Heidelberg, Germany \\ 3 School of Mathematics, University of Edinburgh, Edinburgh EH9 3JZ, Scotland, UK
}

Received 24 November 2006 / Accepted 26 January 2007

ABSTRACT

\begin{abstract}
We investigate the efficiency of galactic mass loss, triggered by ram-pressure stripping and galactic winds of cluster galaxies, on the chemical enrichment of the intra-cluster medium (ICM). We combine $N$-body and hydrodynamic simulations with a semi-numerical galaxy formation model. By including simultaneously different enrichment processes, namely ram-pressure stripping and galactic winds, in galaxy-cluster simulations, we are able to reproduce the observed metal distribution in the ICM. We find that the mass loss by galactic winds in the redshift regime $z>2$ is $\sim 10 \%$ to $20 \%$ of the total galactic wind mass loss, whereas the mass loss by rampressure stripping in the same epoch is up to $5 \%$ of the total ram-pressure stripping mass loss over the whole simulation time. In the cluster formation epochs $z<2$ ram-pressure stripping becomes more dominant than galactic winds. We discuss the non-correlation between the evolution of the mean metallicity of galaxy clusters and the galactic mass losses. For comparison with observations we present two dimensional maps of the ICM quantities and radial metallicity profiles. The shape of the observed profiles is well reproduced by the simulations in the case of merging systems. In the case of cool-core clusters the slope of the observed profiles are reproduced by the simulation at radii below $\sim 300 \mathrm{kpc}$, whereas at larger radii the observed profiles are shallower. We confirm the inhomogeneous metal distribution in the ICM found in observations. To study the robustness of our results, we investigate two different descriptions for the enrichment process interaction.
\end{abstract}

Key words. hydrodynamics - methods: numerical - Galaxy: abundances - galaxies: interactions - galaxies: intergalactic medium galaxies: evolution

\section{Introduction}

From numerous X-ray observations (Fukazawa et al. 1998; Schmidt et al. 2002; Furusho et al. 2003; Sanders et al. 2004; Fukazawa et al. 2004; Hayakawa et al. 2004; Pratt et al. 2007; De Grandi et al. 2004, and Gitti \& Schindler 2004) it is well known that the intra-cluster medium (hereafter ICM) harbours on average $\sim 0.6 \%$ heavy elements in mass, i.e. 0.3 in solar abundance. By using state of the art X-ray space telescopes it was found that the metals are not homogenously distributed within the ICM. Metallicity profiles show a relatively flat distribution in non-cool core clusters, while there is an increase in the central metallicity in cool core clusters (De Grandi et al. 2004; Vikhlinin et al. 2005; Pratt et al. 2007).

Many groups have recently derived detailed two dimensional metallicity maps (e.g. Sanders et al. 2004; Durret et al. 2005; O'Sullivan et al. 2005; Sauvageot et al. 2005; Werner et al. 2006; Sanders \& Fabian 2006; Hayakawa et al. 2006; Finoguenov et al. 2006; Bagchi et al. 2006). All these maps show an inhomogeneous distribution of the heavy elements with several maxima, complex metal patterns and a non-spherically symmetric distribution.

Many processes were investigated to explain the origin of these metals, i.e. galactic winds (De Young 1978), rampressure stripping (Gunn \& Gott 1972), galaxy-galaxy interactions (Gnedin 1998; Kapferer et al. 2005), active galactic nuclei (AGNs, Moll et al. 2007) and intra-cluster supernovae
(Domainko et al. 2004). Ram-pressure stripping, for instance, has been found in several Virgo cluster galaxies by HI observations (Cayatte et al. 1990; Veilleux et al. 1999; Vollmer et al. 1999; Vollmer 2003; Kenney et al. 2004; Vollmer et al. 2004a,b; Koopmann \& Kenney 2004; Crowl et al. 2005; Levy et al. 2006). Galactic winds, driven by supernova explosion within cluster galaxies have been observed and studied in detail in many systems in multiple wavelengths (see reviews by Heckman et al. 2003, and Veilleux et al. 2005). The mass fraction of metals in these outflows depends on galaxy parameters and on environmental conditions. Martin (1999) found that the mass loss by galactic winds scales linearly with the galaxies star formation rate. It was shown, that in the centre of massive clusters the pressure of the ICM can suppress galactic winds (Schindler et al. 2005). Also hydrodynamic simulations of outflows have been performed (Tenorio-Tagle \& Munoz-Tunon 1998; Strickland \& Stevens 2000).

Many groups have investigated the chemical evolution of the ICM using numerical simulations. De Lucia et al. (2004) and Nagashima et al. (2005) studied the enrichment of the ICM with $\mathrm{N}$-body simulations combined with a semi-analytic galaxy formation model. Their conclusion is that nearly all metals found in the ICM are ejected by supernova-driven galactic winds by the most massive galaxies. Additionally, they claim that there is a light chemical evolution since $z=1$. So far, none of these processes alone was sufficient to produce the observed 
pollution of the ICM. Therefore recent simulations were not able to reproduce the observed amount and the spatial distribution of metals in the ICM by galactic winds (Tornatore et al. 2004; Kapferer et al. 2006) or ram-pressure stripping (Domainko et al. 2006). While the effects of galactic winds (e.g. Tornatore et al. 2004, Scannapieco et al. 2006 and Romeo et al. 2006) or rampressure stripping (e.g. Domainko et al. 2006) on galaxies have been studied analytically and numerically by many authors, we focus mainly on the evolution and distribution of the metallicity in the ICM. In this paper we investigate the impact on the enrichment of the ICM by ram-pressure stripping and galactic winds of cluster galaxies. Compared to our previous work (Schindler et al. 2005; Kapferer et al. 2006; Domainko et al. 2006; Moll et al. 2007) we extend this investigation to the epoch where the first galaxies form and compare the efficiency of galactic winds and ram-pressure stripping over a Hubble time.

\section{Simulations}

\subsection{Numerical methods}

As described in Schindler et al. (2005), Kapferer et al. (2006) and Domainko et al. (2006) we apply different code modules to calculate appropriately each of the galaxy-cluster components. The cosmological model used has the following parameters: $\Omega_{\mathrm{m}}=0.3, \Omega_{\Lambda}=0.7, h=0.7, \sigma_{8}=0.93, \Omega_{\mathrm{b}}=0.02 h^{-2}$.

\subsubsection{Dark Matter}

The non-baryonic component is calculated using GADGET2 (Springel 2005) as an $N$-body code, with constrained random fields as initial conditions (Hoffman \& Ribak 1991); implemented by van de Weygaert \& Bertschinger 1996). The $N$-body code provides the underlying evolution of the dark matter (DM) potential for the hydrodynamic code and the dynamically fully described orbits of model cluster galaxies.

\subsubsection{Galaxy formation model}

The properties of the galaxies are calculated by an improved version of the galaxy formation code of van Kampen et al. (1999). The galaxy formation model is semi-numerical in the sense that the merging history of galaxy haloes is taken directly from the cosmological $N$-body simulation. Halo mergers are identified in the $N$-body simulations as events in which a new halo is formed that is both virialised and contains at least two progenitors. We distinguish between major mergers, where two progenitors have at least $30 \%$ of the mass of the merger remnant, and minor mergers, which we treat as accretion events. During any merger event, all galaxies involved undergo a starburst whose intensity depends on the progenitor/merger remnant mass ratio (small for minor mergers), and whose duration depends on the relaxation time of the new halo (short in the case of minor mergers). Galaxy-galaxy mergers are treated differently: all galaxies within a dark matter halo or subhalo suffer from dynamical friction, and will merge with the central galaxy eventually. The dynamical friction time scale is reset after a major merger event (assuming that orbits will be randomized by the event), but not after a minor merger event. When a satellite galaxy merges with the central galaxy of a halo or subhalo, another starburst will occur, using up most of the cold gas left in the satellite. Not all is turned into stars due to feedback, however. In between merger events star formation is quiescent, and we therefore have two types of star formation modes: quiescent star formation in disks with a threshold according to the Kennicutt criterion, and starbursts resulting from major merger events, including halohalo and galaxy-galaxy mergers. Gas that cools within virialised haloes is assumed to settle in a disk with an exponential profile. We use the models of Mo et al. (1998) to set the disk-scale length, with the distinction that we measure the angular momentum of the dark halo from the $N$-body simulation data instead of assuming an analytical model for that. Stellar evolution is modelled using the stellar population synthesis models of Bruzual \& Charlot (2003). The metallicity of the stellar populations affects the cold and hot gas components of a galaxy: a fraction of the metals formed in stars is ejected by $\mathrm{SNe}$ and stellar winds into the surrounding interstellar medium (ISM). The metals that end up in the hot gas component also affect the cooling rate, which depends on metallicity (Sutherland \& Dopita 1993). Chemical evolution is modelled as in Matteucci \& Francois (1989), which includes SN types I and II. The evolution of the metals is followed for the stellar populations as well as for the cold and hot gas reservoirs, and exchanges are also tracked. Material returned to the cold ISM by stellar winds and supernovae has been chemically enriched by the nuclear processes inside the stars.

\subsubsection{Hydrodynamics}

For the treatment of the ICM we use a comoving hydrodynamic code with shock capturing scheme (PPM, Collela \& Woodward 1984), with a fixed mesh refinement scheme (Ruffert 1992) on four levels and radiative cooling (Sutherland \& Dopita 1993). The major improvement with respect to the previously used setup is the usage of comoving coordinates, which was implemented in the hydrodynamic code by applying a operator splitting method. This approach allows us to apply cosmological initial conditions for the hydrodynamic simulation. The largest grid covers a comoving volume of $(20 \mathrm{Mpc})^{3}$. Each finer grid covers $\frac{1}{8}$ of the volume of the next larger grid. With a resolution of $128^{3}$ grid cells on each grid we obtain a finest resolution of $(\sim 19.5 \mathrm{kpc})^{3}$ comoving for each cell on the innermost grid. The $N$-body and hydrodynamics code are calculated starting at $z=40$, while the semi-numerical galaxy formation covers the redshift interval from $z=20$ to $z=0$.

This setup allows us to address questions like:

- what is the efficiency of ram-pressure stripping versus galactic winds as a function of redshift?

- what is the spatial distribution of metals in the ICM ejected by galactic winds and/or lost by ram-pressure stripping?

\subsection{Calculation of the mass loss due to galactic winds}

The mass loss due to galactic winds is calculated using the star formation rate (SFR) $\left(M_{\star}\left[M_{\text {sun }} / \mathrm{yr}\right]\right)$ of a given galaxy, as proposed by Martin (1999). We use the same approach as Springel \& Hernquist (2003), who found that a mass loss due to galactic winds $\dot{M}=\eta M_{\star}$, with a constant fraction $\eta$, is reasonable to fit observations (see Springel \& Hernquist (2003) and references therein). The same was found by Tornatore et al. (2004) who used $\eta=2$. This heuristic approach gives us the mass loss for a given spiral galaxy, which we then add to the hydrodynamic simulation at the galaxy's position. The metals are mixed with the ICM, which is already present in a computational cell. The metallicity of the ejected gas is taken from the semi-numerical galaxy model, which calculates the metals in the hot and cold gas phase by

$\dot{m}_{\mathrm{c}}=Z_{\mathrm{h}} \dot{M}_{\mathrm{c}}-Z_{\mathrm{c}}(1+\beta-R) \Psi_{\mathrm{SF}}+y \Psi_{\mathrm{SF}}$ 
$\dot{m}_{\mathrm{h}}=Z_{h} \dot{M}_{\mathrm{c}}+Z_{\mathrm{c}} \beta \Psi_{\mathrm{SF}}+Z_{\text {Prim }} \dot{M}_{\text {new }}$,

where $Z_{\mathrm{c}}=m_{\mathrm{c}} / M_{\mathrm{c}}\left(Z_{\mathrm{c}}\right.$ the metallcity, $m_{\mathrm{c}}$ the mass of heavy elements and $M_{\mathrm{c}}$ the mass of the cold gas $), Z_{\mathrm{h}}=m_{\mathrm{h}} / M_{\mathrm{h}}\left(Z_{\mathrm{h}}\right.$ the metallcity, $m_{\mathrm{h}}$ the mass of heavy elements and $M_{\mathrm{h}}$ the mass of the hot gas), and $\dot{M}_{\text {new }}$ is the accretion of new baryons by the halo, $\Psi_{\mathrm{SF}}$ is the star formation rate, $Z_{\text {prim }}$ the primordial metallicity and $\beta$ the fraction of supernovae in a stellar population. This results in a lower limit for the metallicity of the galactic-wind ejecta.

\subsection{Calculation of the mass loss due to ram-pressure stripping}

To calculate the mass loss of cluster galaxies due to ram-pressure stripping we follow the classical Gunn \& Gott (1972) criterion. In this model, gas of galactic discs is stripped outside a certain stripping radius where the ram pressure exceeds the restoring gravitational forces. According to Vollmer et al. (2001) the Gunn \& Gott (1972) criterion extended for the inclination angle can explain the HI deficiency of galaxies observed in the region of the Virgo cluster. We use the same approach as presented in detail in Domainko et al. (2006) for sub- and supersonic galaxies interacting with the ICM. The main parameters in the rampressure stripping routine are the relative velocity $v$ of a galaxy with respect to the ICM, the density $\rho_{\text {ICM }}$ of the ICM and the surface density of both, the stellar $M_{\text {star }}$ and the gas $M_{\text {gas }}$ mass in the model galaxy. As shown in Domainko et al. (2006) a certain stripping radius for each galaxy, depending on the properties of the ICM and the galaxy, can be derived

$R_{\text {strip }}=\frac{R_{0}}{2} \times \ln \left(\frac{G M_{\mathrm{star}} M_{\mathrm{gas}}}{v^{2} \rho_{\mathrm{ICM}} 2 \pi R_{0}^{4}}\right)$,

where $R_{0}$ is the disc scale length of the gas distribution in the galaxy. Gaseous matter which is located outside this stripping radius is lost by the galaxy. As a consequence of the used PPM scheme in the grid-based hydrodynamic calculations, shocks induced by merger events and their propagation throughout the cluster are well treated. These shock features have direct impact on the ram-pressure stripping.

\subsection{Combination of galactic winds and ram-pressure stripping}

We combine both enrichment processes in a multi-step approach. First we select from the complete sample of model galaxies the spiral galaxies by requiring a significantly large (i.e. disk scale length larger than bulge radius) star forming, gaseous disk. In a second step the star formation rate in the disk is used to calculate the galactic wind mass loss as described in Sect. 2.2. In order to investigate the relative importance of both processes we study two extreme cases. First we consider a strong influence of ram-pressure stripping on the galactic winds. As long as the galaxy has not fulfilled the criterion for ram-pressure stripping the galaxy is allowed to lose gas by galactic winds. When the galaxy loses matter due to ram-pressure stripping, it will not be affected by galactic winds anymore. This avoids an overestimation of the mass loss by galactic winds, especially in the low redshift regime, i.e. $z<1.5$ where galaxy clusters form and dynamical enrichment processes like ram-pressure stripping become dominant. Numerous observational results point at a quenching of the star formation in galaxies, encountering the dense environment of the ICM (e.g. Haines et al. 2006; Gavazzi et al. 2002).
This description of the combined processes leads to a lower limit in the metal enrichment efficiency for galactic winds at lower redshifts. As a second approach we do not consider any influence of ram-pressure stripping on the galactic winds. A galaxy can have a galactic wind and ram-pressure stripping at the same time. Note that hereafter the index $a$ denotes the first and $b$ denotes the second combination of the two enrichment processes.

\section{The properties of the model galaxy clusters}

In order to investigate the efficiency of different enrichment processes we take a non-cooling flow cluster from a sample of simulated merging systems, which is a lower mass cluster with several strong sub-cluster mergers. The second model cluster is a more massive merging system with nearly more than twice the mass and several minor merger events.

- Model Cluster A: the cluster forms at $z \sim 1.5$ and has two major merger events at $z=0.8$ and $z=0.5$. The final total mass is $1.5 \times 10^{14} M_{\odot}$ in a sphere of radius $1 \mathrm{Mpc}$. We show the X-ray surface brightness and X-ray weighted temperature map at different redshifts in Figs. 1 and 3, respectively.

- Model Cluster B: the formation redshift for this cluster is $z \sim 1.7$. It shows four minor merger events a $z=1.4, z=1.1$, $z=0.5$ and $z=0.3$. The cluster has a final mass of $3.4 \times$ $10^{14} M_{\odot}$ within a sphere of radius $1 \mathrm{Mpc}$. We show the Xray surface brightness and X-ray weighted temperature map at different redshifts in Figs. 2 and 4, respectively.

\section{Results}

\subsection{X-ray weighted maps}

In order to compare the model clusters with observations we extract X-ray surface brightness, X-ray weighted temperature and metal maps at different redshifts. In Fig. 1 X-ray surface brightness maps for model cluster $A$ at redshifts $z=1,0.7,0.5$ and 0 are presented on a logarithmic scale. The area covers $(2.5 \mathrm{Mpc})^{2}$ in comoving space. The final X-ray surface brightness map has a drop of $\sim 2$ mag from the cluster centre to $r_{200}$, which is consistent with observations of non-cooling flow clusters (Arnaud et al. 2002; De Filippis et al. 2003). The hierarchical formation of the cluster is clearly visible, especially at redshift 1 , when the cluster is forming. At lower redshifts model cluster A shows an asymmetric shape, which is a common feature for non cool-core clusters, that typically undergo several mergers. The model cluster begins to relax at redshift $z=0$, nevertheless the spherical shape has not fully established yet.

In Fig. 2 the X-ray surface brightness maps for model cluster $\mathrm{B}$ at different redshifts $(z=1,0.7,0.5$ and 0$)$ are presented. In contrast to model cluster $A$, this cluster shows a rather relaxed shape already at $z=0.7$.

In Figs. 3 and 4 we show X-ray weighted temperature maps for the model clusters at the same redshifts as in Figs. 1 and 2. The hierarchical growth of the model cluster A, which is a direct consequence of the initial power spectrum, is visible. After the formation along a filamentary structure, the cluster builds up via several mergers, which leads to the complex dynamical state of the gas. At the present epoch the cluster has a distinct temperature distribution, see the $2.5 \mathrm{Mpc}$ comoving maps, with a drop towards the cluster outskirts in the order of 50\%. Model cluster B shows a common feature of relaxed clusters, namely a drop in temperature towards the innermost region, i.e. a cool core. As we are not interested in the gas dynamics of the cooling flow 


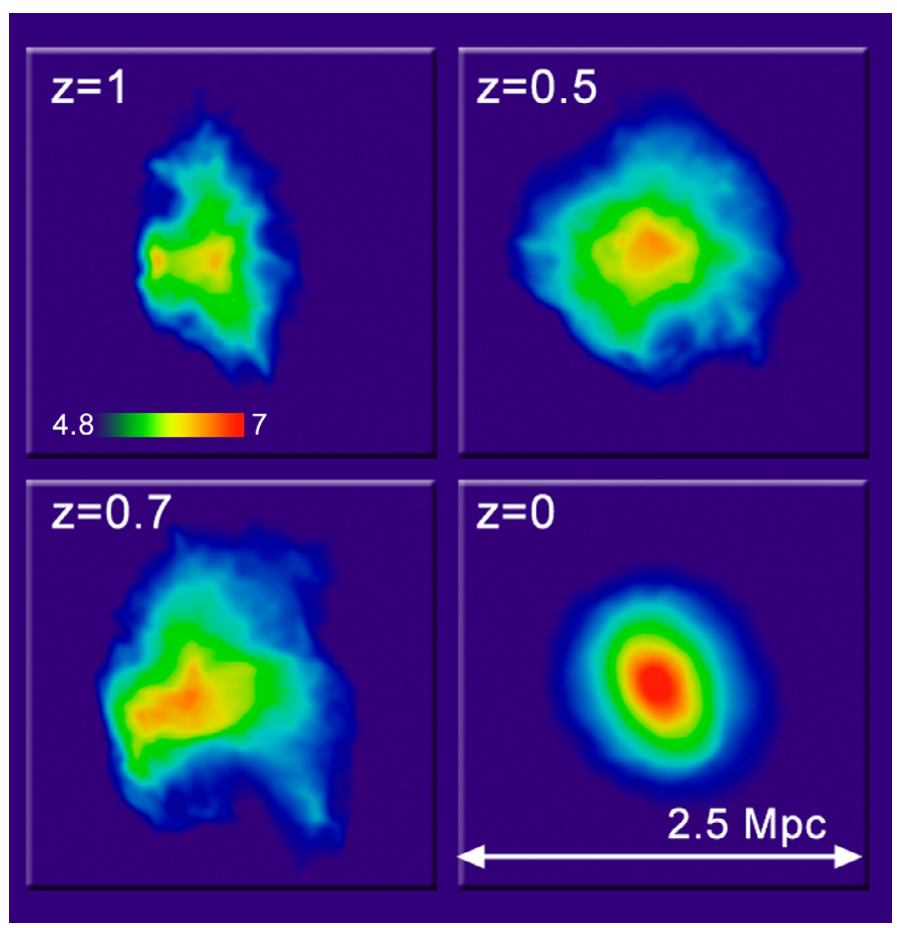

Fig. 1. X-ray surface brightness maps for different redshifts $(z=1,0.7$, 0.5 and 0 ) for model cluster A. The maps have $2.5 \mathrm{Mpc}$ comoving on a side and give the $\mathrm{X}$-ray emission in a logarithmic scale.

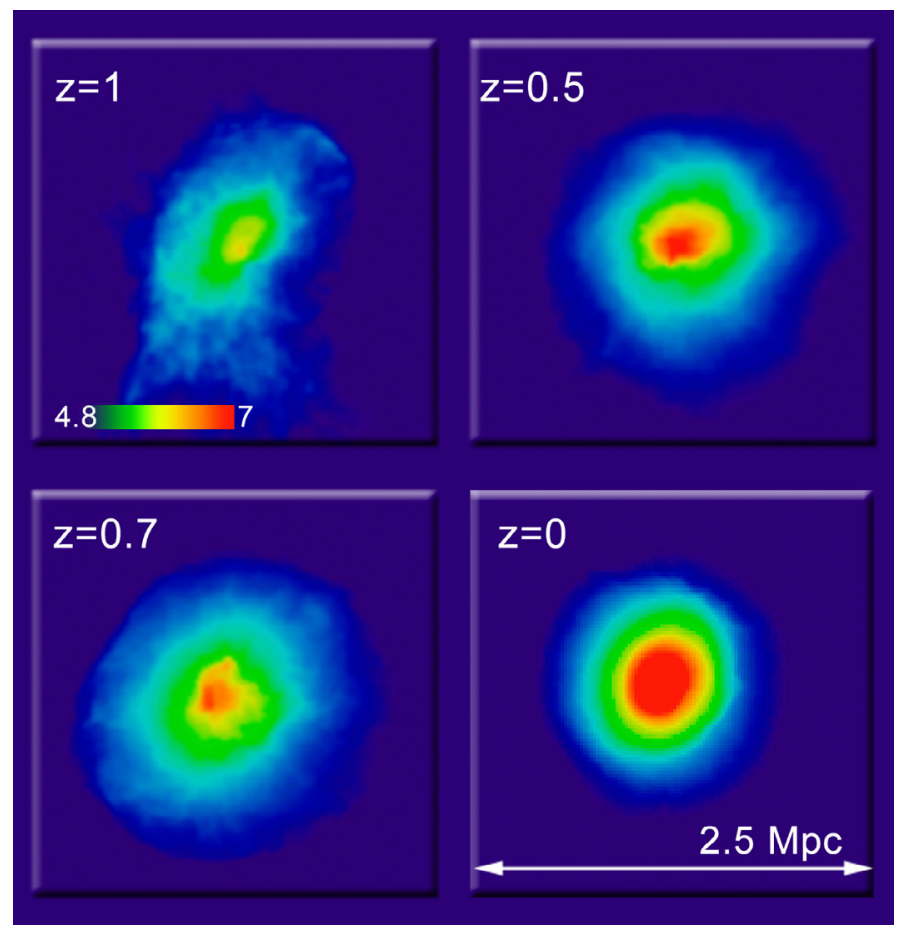

Fig. 2. X-ray surface brightness maps for different redshifts $(z=1,0.7$, 0.5 and 0 ) for model cluster $\mathrm{B}$. The maps have $2.5 \mathrm{Mpc}$ comoving on a side and give the $\mathrm{x}$-ray emission in a logarithmic scale.

region, but rather in the large-scale distribution of the metals in the ICM, we do not introduce a heating source to counterbalance the overcooling. However, due to our limited spatial resolution we underestimate the cooling in this region, avoiding a cooling catastrophe. Hence, except of the innermost computational cells,

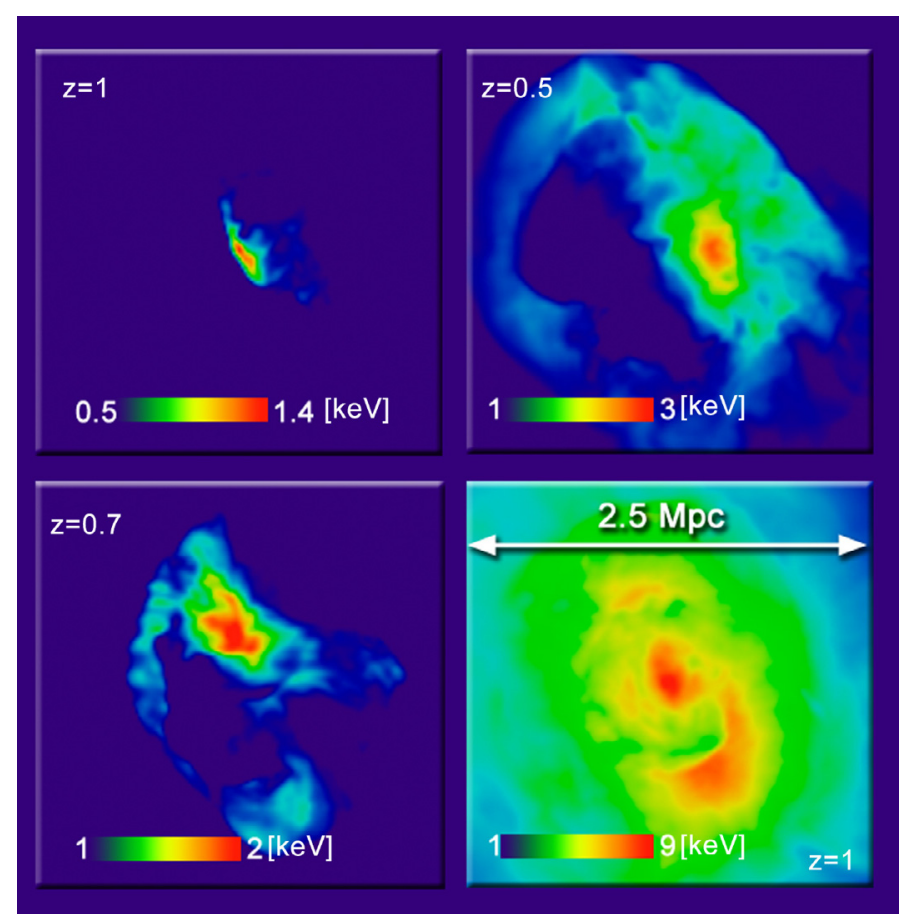

Fig. 3. X-ray weighted temperature maps for different time steps $(z=1$, $0.7,0.5$ and 0 ) for model cluster A. The maps have a size of $2.5 \mathrm{Mpc}$ comoving and give the temperature in $\mathrm{keV}$ linear scale.

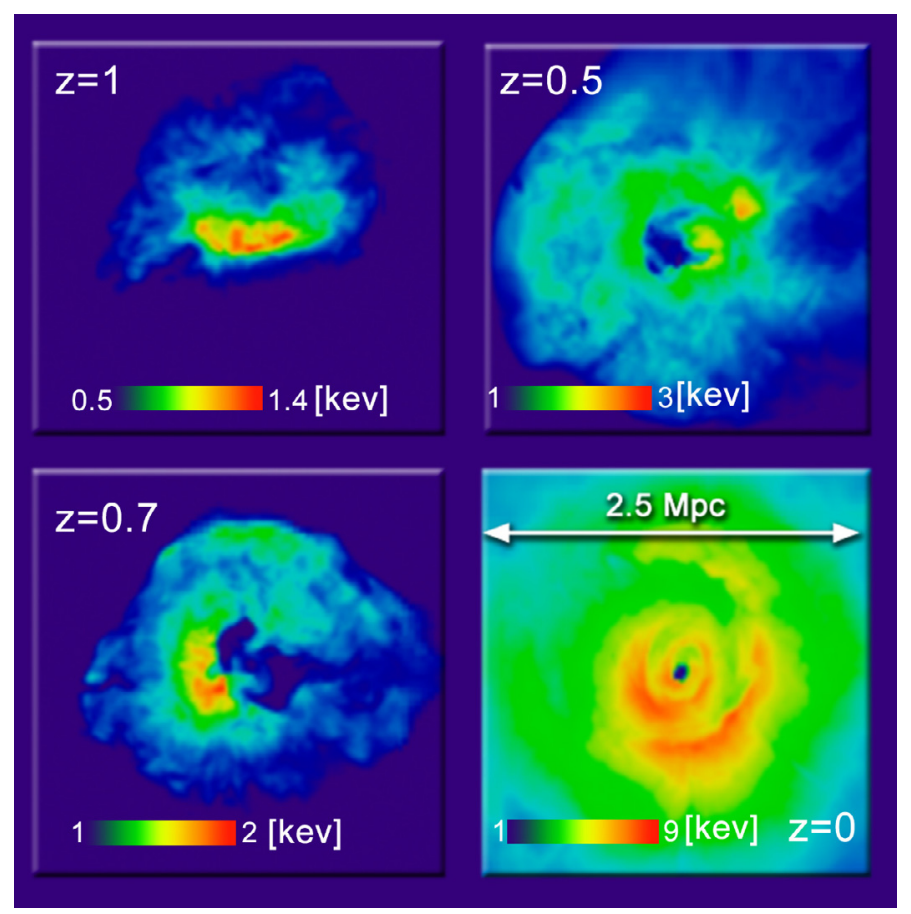

Fig. 4. X-ray weighted temperature maps for different time steps $(z=1$, $0.7,0.5$ and 0 ) for model cluster $\mathrm{B}$. The maps have a size of $2.5 \mathrm{Mpc}$ comoving and give the temperature in $\mathrm{keV}$ linear scale.

the radial temperature profile resembles that of observed relaxed galaxy clusters.

In order to investigate the strength of the different enrichment processes and the spatial distribution of the metals, we construct X-ray weighted metal maps from our 3D data. These maps can be compared to observations. In Figs. 5 and 6 metallicity maps of the two model clusters for the different 


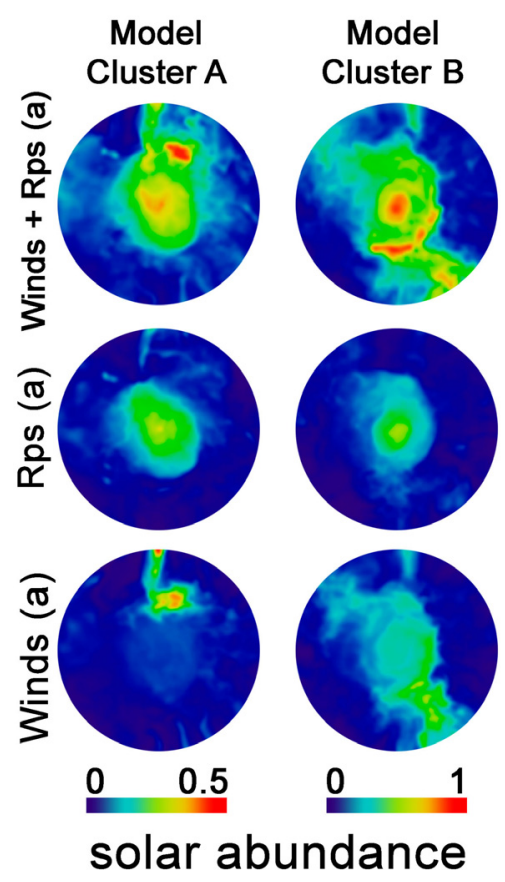

Fig. 5. X-ray weighted metal maps for the two model clusters in an area with $2000 \mathrm{kpc}$ diameter for different enrichment processes at redshift 0 , enrichment process combination $a$.

enrichment process combinations descriptions $a$ and $b$ are presented. We construct them for redshift $z=0$ within an area of $2 \mathrm{Mpc}$ in diameter. The left column corresponds to the merging model cluster A, whereas the right column shows the relaxed system of model cluster B. In order to highlight the different spatial distribution of the metals emerging from the two different enrichment processes, i.e. ram-pressure stripping and galactic winds, we show separate metal maps for each enrichment process along with a map that gives the overall metal distribution.

\subsection{Mass loss by ram-pressure stripping and galactic winds}

To address the question on the different efficiencies of the two enrichment processes, we give in Tables 1 and 2 the integrated mass loss for the cluster galaxies in two different redshift regimes for ram-pressure stripping and galactic winds for enrichment process combination $a$ and $b$. As the clusters form at redshifts $z<2$ and because ram-pressure stripping depends highly on the density of the environment, it is more important in this redshift regime. In the case of enrichment combination $b$ and model cluster B ram-pressure stripping is less important for enriching the ICM. As galactic winds are not suppressed by rampressure stripping or the pressure of the ICM they nearly exhaust the gas reservoir of the galaxies. Galactic winds, on the contrary, induce higher mass losses at higher redshifts. The mass loss by this process is mainly driven by the supernova rate of a system, which is tightly linked to the star formation rate. As the star formation rate peaks at redshifts larger than 2 (e.g. Hopkins 2004), the mass loss of galactic winds is the more dominant process for the removal of gas from galaxies in this redshift regime. Rampressure stripping is in terms of total mass loss a factor of three more efficient than winds in model cluster $\mathrm{A}$ and $50 \%$ in model cluster B for the enrichment process combination $a$. This can be explained by the merger events in model cluster A, which introduce disturbances in the ICM. These increase the ram pressure onto the cluster galaxies. In model cluster B the difference of

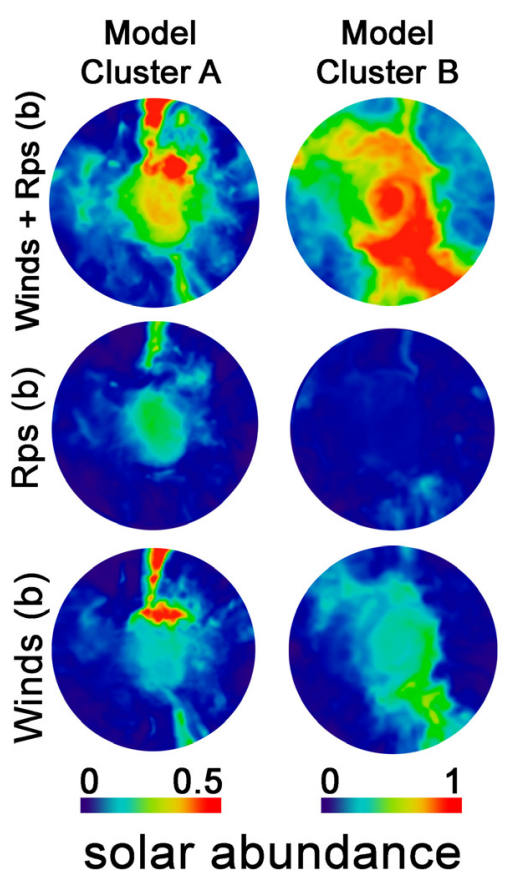

Fig. 6. X-ray weighted metal maps for the two model clusters in an area with $2000 \mathrm{kpc}$ diameter for different enrichment processes at redshift 0 , enrichment process combination $b$.

Table 1. Fractions of the integrated mass loss due to galactic winds for model cluster A and B.

\begin{tabular}{cccc}
\hline \hline Model & Redshift & Winds $a$ & Winds $b$ \\
\hline A & $z>2$ & $20.5 \%$ & $13.5 \%$ \\
A & $z<2$ & $79.5 \%$ & $87.5 \%$ \\
A & wind mass loss & $1.2 \times 10^{11} M_{\odot}$ & $2.5 \times 10^{11} M_{\odot}$ \\
\hline B & $z>2$ & $21.4 \%$ & $12.3 \%$ \\
B & $z<2$ & $78.6 \%$ & $87.7 \%$ \\
B & wind mass loss & $4.4 \times 10^{11} M_{\odot}$ & $1.7 \times 10^{12} M_{\odot}$ \\
\hline
\end{tabular}

Total mass loss (galactic winds and ram-pressure stripping) model cluster A enrichment combination $a$ : $4.8 \times 10^{11} M_{\odot}$, model cluster B: $1.12 \times 10^{12} M_{\odot}-$ enrichment combination $b: 5.6 \times 10^{11} M_{\odot}$, model cluster B: $1.9 \times 10^{12} M_{\odot}$ in a $(5 \mathrm{Mpc})^{3}$ comoving volume.

mass loss between ram-pressure stripping and galactic winds is lower compared to model cluster A. Compared to the merging system, where also in the outer regions of the cluster galaxies are affected by ram-pressure, in relaxed systems ram-pressure is predominately present in the central cluster regions and therefore not so efficient. In the case of enrichment process combination $b$ the ratio of the mass loss between ram-pressure stripping and galactic winds is comparable to enrichment process combination $a$ for model cluster A. Model cluster B shows a different picture, mainly because enrichment process combination $b$ does deplete remove too much gas from the galaxies by galactic winds, leading to a small amount gas left in the galaxies at the epochs when ram-pressure stripping becomes more important. It seems that in this case we underestimate the interaction between ram-pressure stripping and galactic winds.

In Fig. 7 the mass loss of all galaxies due to ram-pressure stripping in a $(5 \mathrm{Mpc})^{3}$ cube for both enrichment process description is presented. As ram-pressure stripping is strongly dependent on the environment the mass loss increases as the cluster forms, i.e. in the redshift regime $z<2$. In addition merging events increase the density in the ICM temporarily, leading to 
Table 2. Fractions of the integrated mass loss due to ram-pressure stripping for model cluster $\mathrm{A}$ and $\mathrm{B}$.

\begin{tabular}{cccc}
\hline \hline Model & Redshift & RPS $a$ & RPS $b$ \\
\hline A & $z>2$ & $2.7 \%$ & $4.7 \%$ \\
A & $z<2$ & $97.3 \%$ & $95.3 \%$ \\
A & rps mass loss & $3.6 \times 10^{11} M_{\odot}$ & $3.1 \times 10^{11} M_{\odot}$ \\
\hline B & $z>2$ & $4.8 \%$ & $42.4 \%$ \\
B & $z<2$ & $95.2 \%$ & $57.6 \%$ \\
B & rps mass loss & $6.8 \times 10^{11} M_{\odot}$ & $1.7 \times 10^{11} M_{\odot}$ \\
\hline
\end{tabular}

Total mass loss (galactic winds and ram-pressure stripping) model cluster A enrichment combination $a: 4.8 \times 10^{11} M_{\odot}$, model cluster B: $1.12 \times 10^{12} M_{\odot}-$ enrichment combination $b: 5.6 \times 10^{11} M_{\odot}$, model cluster B: $1.9 \times 10^{12} M_{\odot}$ in a $(5 \mathrm{Mpc})^{3}$ comoving volume. RPS... Ram-Pressure Stripping.

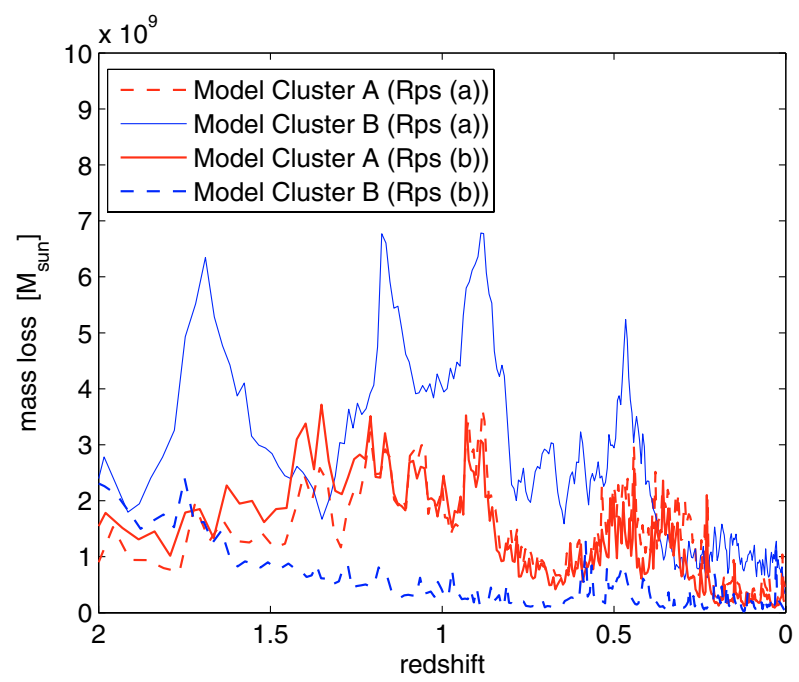

Fig. 7. Mass loss due to ram-pressure stripping as a function of redshift for the two model clusters, in a $5 \mathrm{Mpc}$ on a side cube around the cluster centre, for two different enrichment process descriptions.

strong mass losses of galaxies passing this regions. Additionally, groups of galaxies which belong to sub-clusters and enter the main cluster, fall into the cluster with high velocities and therefore increase the ram pressure. This again leads to temporarily high mass losses. Although the massive model cluster B show no major merger events, ram-pressure stripping is even more efficient than in the merging model cluster A for the enrichment process combination $a$. Due to the higher mass of model cluster B (more than twice as massive than cluster A), more massive galaxies which harbour more gas mass in the discs reside in it. As in addition the density in the cluster's central regions is higher the mass loss is larger in model cluster B.

Figure 8 gives the mass loss of all galaxies in a $(5 \mathrm{Mpc})^{3}$ cube for the galaxies for both enrichment process combinations due to galactic wind. The increase of mass loss by galactic winds at redshift $z \sim 2$ in model cluster B has two reasons. There is an obvious connection between the mass loss efficiency of galactic winds and ram-pressure stripping and the formation history of a galaxy cluster. If the system is a merging system (several major events at all epochs) then the differences in mass loss between galactic winds and ram-pressure are in the order of several $100 \%$, resulting in ram-pressure stripping as the dominant enrichment process for both enrichment process combinations. In relaxed, massive systems the ratio drops significantly, for model cluster B ram-pressure stripping is only $50 \%$ more efficient for

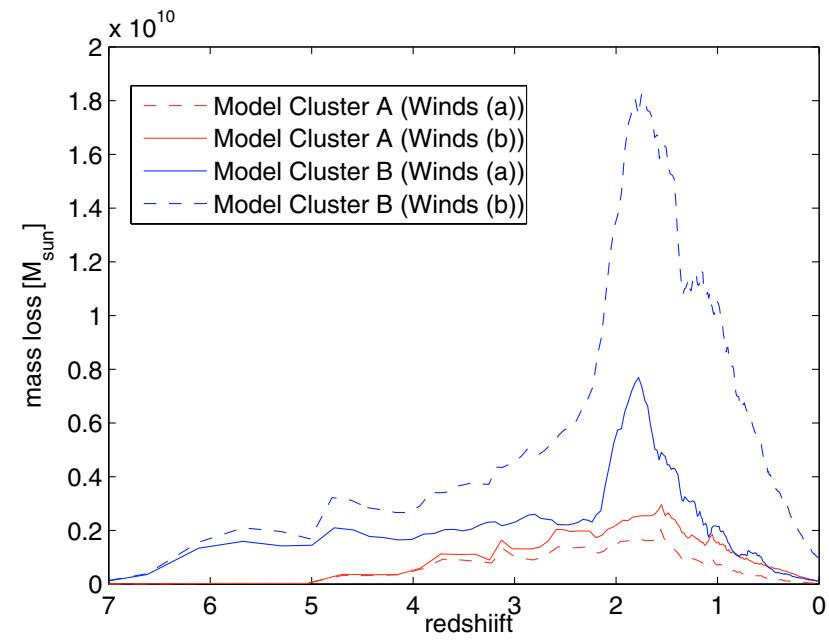

Fig. 8. Mass loss due to galactic winds as a function of redshift for the two model clusters, in a cube around the cluster centre with $5 \mathrm{Mpc}$ on a side, for two different enrichment process descriptions. Note that enrichment process description $b$ has higher mass losses, as there is no feedback of ram-pressure stripping on the galactic winds.

enrichment process combination $a$. The enrichment combination $b$ leads to a contrary picture. In this case the mass loss by galactic winds is an order of magnitude higher than due to rampressure stripping. The amount of matter lost by cluster-galaxies is a strong function of the number of cluster galaxies. As a more massive system has more galaxies the absolute value of mass loss is higher than in the merging system. To what extent these mass losses enrich the ICM will be discussed in the next section.

\subsection{Metallicity profiles}

Observers often give metallicity profiles of galaxy clusters. By integrating X-ray events in radial bins it is possible to construct $\mathrm{X}$-ray spectra to investigate metal abundances in a given annulus. With a longer exposure time or for a higher luminosity of the galaxy cluster, more events by X-ray photons arrive at the detector. This makes it possible to increase the resolution of the metallicity profiles or to construct 2D maps of the metallicity of the observed galaxy cluster. To compare our simulated ICM properties with observations we provide metal profiles and X-ray weighted metallicity maps.

The evolution of the mean metallicity in an area with $500 \mathrm{kpc}$ physical radius around the cluster centre is shown for both model clusters and enrichment process combinations in Fig. 9. At $z<$ 1.2 there is a clear difference in metallicity present, which belongs to the different formation epoch for the both clusters. The relaxed system has already assembled, leading to higher metallicities at $z=1.2$. As metallicity is a measure of heavy elements to primordial the merging system, building up by mergers introduces enriched ICM to the cluster region at all epochs, leading to a slightly increase in the mean metallicity. Model cluster B is has nearly constant mean metallicities in the redshift regime $z<1.2$. The difference is given by the different amount of primordial ICM mass accretion in the same interval. Whereas the enrichment combinations $a$ and $b$ deliver nearly the same results for model cluster A, there is a difference for model cluster B. In this case nearly all metals are lost by galactic winds, as they are not suppressed by any physical process like pressure of the ICM onto the galaxies (Schindler et al. 2005). 


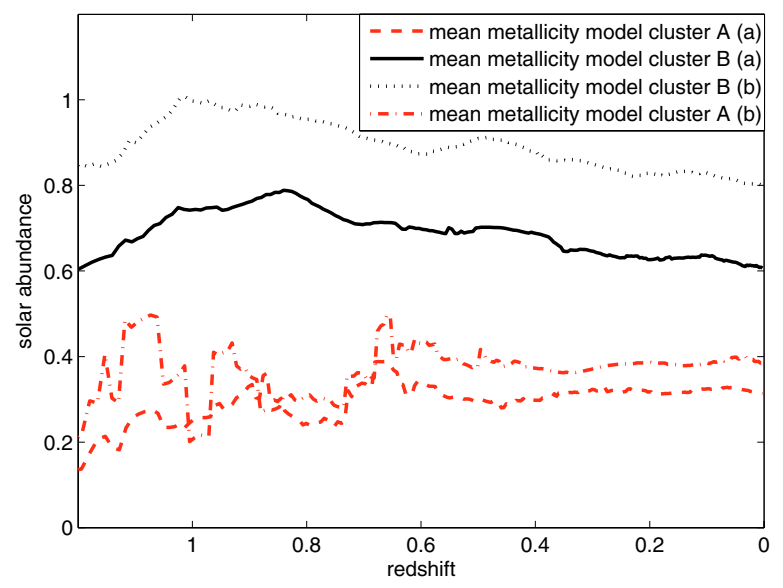

Fig. 9. Evolution of the mean metallicity for both model clusters taken from the X-ray emission within a circle of $500 \mathrm{kpc}$ radius for both enrichment process combinations $a$ and $b$.

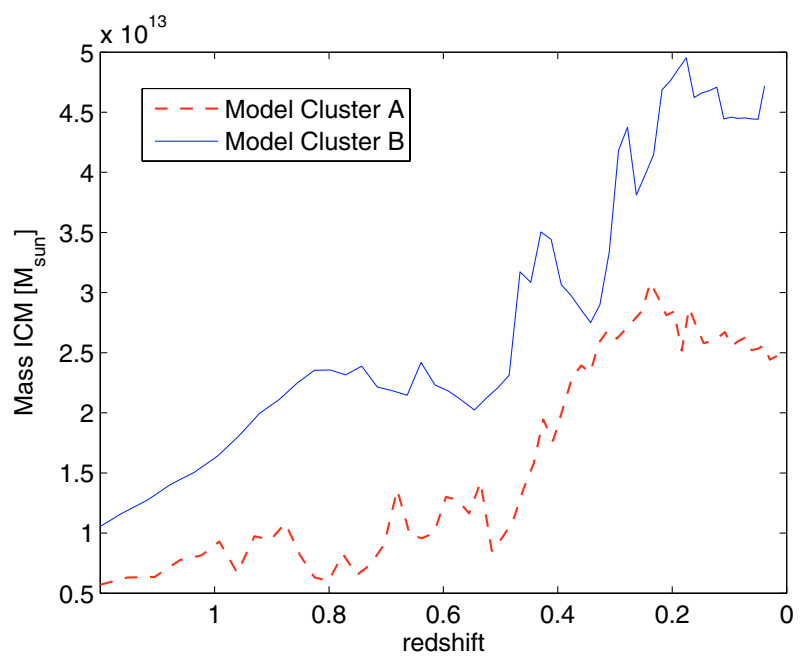

Fig. 10. Evolution of the ICM mass in a sphere with 2 Mpc radius around the cluster centre.

Figure 10 shows the evolution of the ICM mass within a sphere with a radius of $2 \mathrm{Mpc}$ comoving around the cluster centre. Besides the strong signs of sub-clusters and groups falling into the sphere, the main difference between model cluster A and $\mathrm{B}$ is the growth of mass between $z=1.2$ and $z=0$. Metals ejected at higher redshift and falling towards the cluster centre have to mix with yet unpolluted gas, resulting in a decrease of metallicity in model cluster B. In model cluster A the amount of ICM in the same redshift interval does not increase so much, despite during merger events. Therefore the metals ejected by galactic winds and removed by ram-pressure stripping do increase the overall metallicity in the $500 \mathrm{kpc}$ radius area around the cluster centre. This slight increase is consistent with observations, e.g. Balestra et al. (2007). In the case of the massive model cluster B this seems not to be the case. Combining different clusters with different luminosities might explain this discrepancy.

In Fig. 11 observed metallicity profiles for six different noncooling flow clusters are shown. The data points are taken from Pratt et al. (2007) (RX J0003+0203, RX J0020-2542, RX J05473152, RX J1516+0005 and RX J2023-2056) and Hayakawa et al. (2006) (A1060), as they present non-cooling flow clusters.

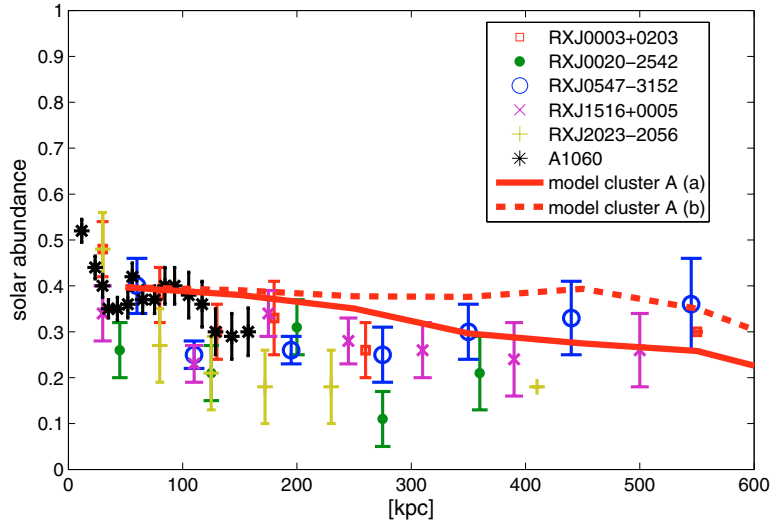

Fig. 11. Metallicity profiles for six non-cooling flow clusters and model cluster A for both enrichment process combinations $a$ and $b$.

A common striking feature for all measured radial metallicity profiles is a nearly flat distribution within a radius of $600 \mathrm{kpc}$ around the cluster centre. In contrast to cooling flow clusters, which show an enhancement of metallicities towards the cluster centre, e.g. De Grandi et al. (2004), non-cooling flow clusters have typical mean metallicities in the order of $0.3-0.5$ in solar abundance in the central region. We include in Fig. 11 the metallicity profiles for model cluster A with both enrichment process combinations $a$ and $b$. We are able to reproduce both the observed spatial distribution and the observed amount of metals when taking into account both enrichment processes. Due to the limited numerical resolution, we are not able to follow the increase in the observed profiles in the very central $(\sim 50 \mathrm{kpc})$ region of the clusters. By taking into account two enrichment processes, namely galactic winds and ram-pressure stripping, the profiles show the relatively flat behaviour over large radii.

In Fig. 12 the metallicity profiles for the metals, lost by galactic winds and by ram-pressure stripping in model cluster A for both enrichment processes combinations $a$ and $b$ are presented. Galactic winds are present at all radii, ram-pressure stripping is more concentrated towards the centre. This would in principle lead to different element ratios at radii below and above $\sim 500 \mathrm{kpc}$, which should be present in very long exposure X-ray observations.

Figure 13 shows the different profiles (ram-pressure stripping, galactic winds and both) of model cluster B for enrichment process combination $a$ as well as a mean profile for cool-core clusters from De Grandi et al. (2004). In the case of massive clusters, galactic winds are more efficient at radii grater than $300 \mathrm{kpc}$, whereas ram-pressure stripping enriches the cluster centre more efficiently. The flat distribution present in the non-cooling flow systems alters to a distribution clearly increasing towards the centre. The simulation overestimates the observed metallicity. The slope of the observed iron profile is reproduced by the simulation out to $400 \mathrm{kpc}$. Above $400 \mathrm{kpc}$ the slope is too steep, compared to observations. The enrichment process combination $b$ gives a different result, leading to very small mass loss by rampressure stripping.

\section{Discussion and conclusions}

We investigate the efficiency of galactic winds and ram-pressure stripping in combined $N$-body/hydrodynamic simulations of the intra-cluster medium with semi-numerical galaxy modelling. The terminus mass loss in our simulations refers to galactic 


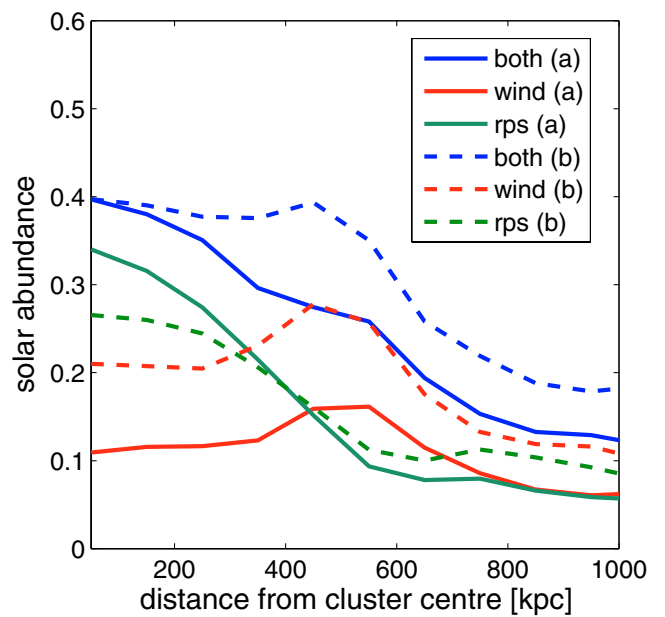

Fig. 12. Metallicity profiles of the model clusters, presented in Figs. 5 and 6 . The profiles for the metals ejected by galactic winds, rampressure stripping and both enrichment precesses together for the two enrichment process combinations $a$ and $b$ are shown.

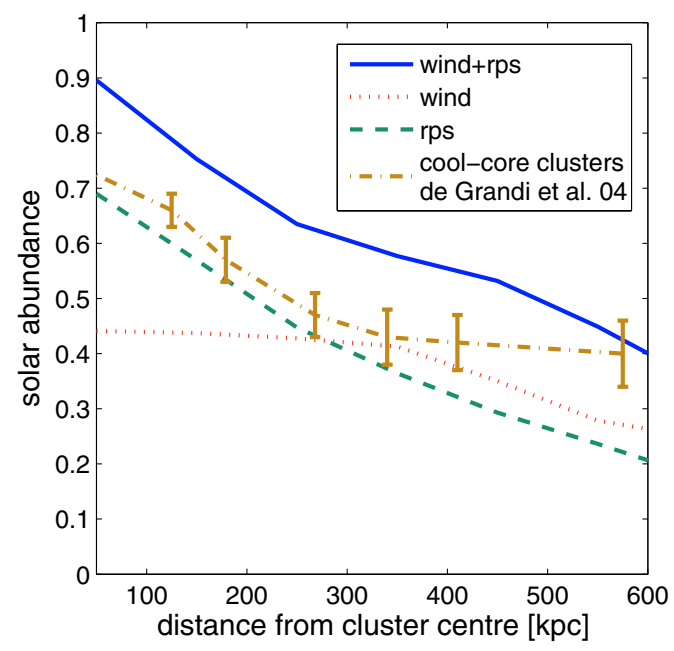

Fig. 13. Metallicity profiles of the model clusters, presented in Fig. 5. The profiles for the metals ejected by galactic winds, ram-pressure stripping and both enrichment processes for enrichment process combinations $a$ are shown. The dashed-dotted line corresponds to the mean of cool-core clusters presented in De Grandi et al. (2004).

material, which is not gravitationally bound to a galaxy anymore. From the simulations we calculate X-ray weighted metallicity maps and profiles as well as mass loss histories for the model cluster galaxies. The profiles are compared with observations. A major uncertainty are the metallicities of the ejecta and stripped gas of the galaxies. This leads in our setup to higher or lower overall metallicities in the ICM, but would not affect the spatial distribution of metals in the ICM. Given the model assumptions, we find the following

1. We confirm the inhomogeneous distribution of heavy elements found in the intra-cluster medium.

2. The mass loss due to galactic winds is half the mass loss caused by ram-pressure stripping in the case of a merging galaxy cluster if ram-pressure stripping is allowed to suppress the outflow by galactic winds. In the case of no influence of ram-pressure stripping onto galactic winds the mass loss by galactic winds is $80 \%$ of the mass loss by ram-pressure stripping. In the case of a relaxed, massive system the mass loss by ram-pressure stripping is three times higher than the mass loss by galactic winds if ram-pressure stripping suppresses the outflow of galactic winds. In this case ram-pressure stripping is the dominating enrichment process in cool-core galaxy clusters. If galactic winds are not affected by ram-pressure stripping the efficiency of both processes permute, leading to nearly no mass loss by ram-pressure stripping.

For merging galaxy clusters the number of galaxies losing mass by ram-pressure stripping is nearly as high as in a relaxed system. This difference can be explained by subcluster merger events, increasing the density of the ICM and the relative velocities of the galaxies with respect to the ICM.

3. Whereas galactic winds trigger most of the mass loss in the redshift regime $z>2$, ram-pressure stripping increases the mass loss in the redshift interval $z<2$, in which the clusters start to form.

4. We traced the evolution of the metallicity in the ICM and found an increase of the metallicity in a $1 \mathrm{Mpc}$ area around the cluster centre in the redshift regime $z<1.2$ for the merging system. The relaxed, massive galaxy cluster shows nearly a constant metallicity in the same redshift regime. Unpolluted ICM entering the cluster region and the different efficiency of the enrichment processes as a function of redshift explains this behaviour. In the case of the merging system enrichment processes are relatively more efficient over all redshifts, leading to a slight increase for the mean metallicity. In the relaxed system ram-pressure stripping and galactic winds do not enrich unpolluted ICM to such an amount that the metallicity of the whole cluster increases.

5. Comparing the metallicity profiles of observed merging clusters with model clusters, we find good agreement in the spatial distribution of the metallicity for merging galaxy clusters. In the case of relaxed, massive systems the slope of the observed metallicity profile is well reproduced in the central $\sim 300 \mathrm{kpc}$, whereas beyond $\sim 300 \mathrm{kpc}$ the observed profiles are shallower.

6. A comparison of the metals lost by galactic winds and rampressure stripping leads to the conclusion that at radii less than $500 \mathrm{kpc}$ matter lost by ram-pressure stripping dominates the profiles, whereas in the outer regions galactic winds contribute to the same amount. If galactic winds are not affected by ram-pressure stripping, galactic winds are more efficient over the whole galaxy cluster in the case of a massive system. If distinct metal blobs caused by galaxies with strong galactic winds fall towards the cluster centre, humps in the metallicity profiles are visible (see Figs. 5, 6 and 12).

Acknowledgements. The authors are grateful to the anonymous referee for his/her fruitful comments that helped to improve the paper. The authors would like to thank Volker Springel for providing GADGET2 and Paul Ricker for clarifying explanations. Edmund Bertschinger and Rien van de Weygaert are acknowledged for providing their constrained random field code. The authors acknowledge the Austrian Science Foundation (FWF) through grant P18523-N16 and grant P18416-N16, the German Science Foundation (DFG) through grant $\mathrm{Zi} \mathrm{663/6-1.} \mathrm{Thomas} \mathrm{Kronberger} \mathrm{is} \mathrm{a} \mathrm{recipient} \mathrm{of} \mathrm{a} \mathrm{DOC-fellowship} \mathrm{of}$ the Austrian Academy of Science. Magdalena Mair is a recipient of a Doktoratsstipendium der Universität Innsbruck and a fellowship of Mils 
Electronic. In addition, the authors acknowledge the ESO Mobilitätsstipendien des bm:bwk (Austria), the Tiroler Wissenschaftsfonds (Gefördert aus Mitteln des vom Land Tirol eingerichteten Wissenschaftsfonds), the UniInfrastrukturprogramm 2005/06.

\section{References}

Arnaud, M., Aghanim, N., \& Neumann, D. M. 2002, A\&A, 389, 1 Bagchi, J., Durret, F., Neto, G. B. L., \& Paul, S. 2006, Science, 314, 791 Balestra, I., Tozzi, P., Ettori, S., et al. 2007, A\&A, 462, 429

Bruzual, G., \& Charlot, S. 2003, MNRAS, 344, 1000

Cayatte, V., van Gorkom, J. H., Balkowski, C., \& Kotanyi, C. 1990, AJ, 100, 604 Collela, P., \& Woodward, P. R. 1984, J. Comp. Phys., 54, 174

Crowl, H. H., Kenney, J. D. P., van Gorkom, J. H., \& Vollmer, B. 2005, AJ, 130, 65

De Filippis, E., Schindler, S., \& Castillo-Morales, A. 2003, A\&A, 404, 63 De Grandi, S., Ettori, S., Longhetti, M., \& Molendi, S. 2004, A\&A, 419, 7 De Lucia, G., Kauffmann, G., \& White, S. D. M. 2004, MNRAS, 349, 1101 De Young, D. S. 1978, ApJ, 223, 47

Domainko, W., Gitti M., Schindler, S., \& Kapferer, W. 2004, A\&A, 425, L21

Domainko, W., Mair, M., Kapferer, W., et al. 2006, A\&A, 452, 795

Durret, F., Lima Neto, G. B., \& Forman, W. 2005, A\&A, 432, 809

Finoguenov, A., Henriksen, M. J., Miniati, F., Briel, U. G., \& Jones, C. 2006, ApJ, 643, 790

Fukazawa, Y., Makishima, K., Tamura, T. et al. 1998, PASJ, 50, 187

Fukazawa Y., Kawano N., Kawashima K. 2004, ApJ, 606, L109

Furusho T., Yamasaki N. Y., Ohashi T. 2003, ApJ, 596, 181

Gavazzi, G., Boselli, A., Pedotti, P., Gallazzi, A., \& Carrasco, L. 2002, A\&A, 396,449

Gnedin, N. Y. 1998, MNRAS, 294, 407

Gunn, J. E., \& Gott J. R.III, 1972, ApJ, 176, 1

Gitti, M., \& Schindler, S. 2004, A\&A, 427, L9

Haines, C. P., Merluzzi, P., Mercurio, A., et al. 2006, MNRAS, 371, 55

Hayakawa, A., Furusho, T., Yamasaki, N. Y., Ishida, M., \& Ohashi, T. 2004, PASJ 56, 743

Hayakawa, A., Hoshino, A., Ishida, M., et al. 2006, PASJ, 58, 695

Heckman, T. M. 2003, Rev. Mex. Astron. Astrofis. Conf. Ser., 17, 47

Hoffman Y., \& Ribak, E. 1991, ApJ, 380, L5

Hopkins, A. M. 2004, ApJ, 615, 209

van Kampen, E., Jimenez, R., Peacock, J. A. 1999, MNRAS, 310, 43

Kapferer, W., Knapp, A., Schindler, S., Kimeswenger, S., \& van Kampen, E. 2005, A\&A, 438, 87

Kapferer, W., Ferrari, C., Domainko., W., et al. 2006, A\&A, 447, 827
Kenney, J. D. P., van Gorkom, J. H., \& Vollmer, B. 2004, AJ, 127, 3361

Koopmann, R. A., \& Kenney, J. D. P. 2004, ApJ, 613, 866

Levy, L., Rose, J. A., van Gorkom, J. H., \& Chaboyer, B. 2006, AJ, accepted [arXiv: astro-ph/0611591]

Matteucci, F., \& Francois, P. 1989, MNRAS, 239, 885

Martin, C. L. 1999, ApJ, 513, 156

Mo, H. J., Mao, S., \& White, S. D. M. 1998, MNRAS, 295, 319

Moll, R., Schlindler, S., Domainko, W., et al. 2007, A\&A, 463, 513

Nagashima, M., Lacey, C. G., Okamoto, T., et al. 2005, MNRAS, 363, L31

O'Sullivan, E., Vrtilek, J. M., Kempner, J. C., David, L. P., \& Houck, J. C. 2005, MNRAS, 357, 1134

Pratt, G. W., Boehringer, H., Croston, J. H., et al. 2007, A\&A, 461, 71

Romeo, A. D., Sommer-Larsen, J., Portinari, L., \& Antonuccio-Delogu, V. 2006, MNRAS, 371, 548

Ruffert, M. 1992, A\&A, 265, 82

Sanders, J. S., \& Fabian, A. C. 2006, MNRAS, 371, 1483

Sanders J. S., Fabian A. C., Allen S., Schmidt R. W. 2004, MNRAS, 349, 952

Sauvageot, J. L., Belsole, E., \& Pratt, G. W. 2005, A\&A, 444, 673

Scannapieco, C., Tissera, P. B., White, S. D. M., \& Springel, V. 2006, MNRAS, 371,1125

Schindler, S., Kapferer, W., Domainko, W., et al. 2005, A\&A, 435, L25

Schmidt R. W., Fabian A. C., Sanders, J. 2002 MNRAS, 337, 71

Springel, V. 2005, MNRAS, 364, 1105

Springel, V., \& Hernquist, L. 2003, MNRAS, 339, 289

Strickland, D. K., \& Stevens, I. R. 2000, MNRAS, 314, 511

Sutherland, R. S., \& Dopita, M. A. 1993, ApJS, 88, 253

Tenorio-Tagle, G., \& Munoz-Tunon, C. 1998, MNRAS, 293, 299

Tornatore, L., Borgani, S., Matteucci, F., Recchi, S., \& Tozzi, P. 2004, MNRAS, 349, L19

van de Weygaert R., Bertschinger E. 1996, MNRAS, 281, 84

Veilleux, S., Bland-Hawthorn, J., Cecil, G., Tully, R. B., \& Miller, S. T. 1999, ApJ, 520, 111

Veilleux, S., Cecil, G., \& Bland-Hawthorn, J. 2005, ARA\&A, 43, 769

Vikhlinin, A., Markevitch, M., Murray, S. S., et al. 2005, ApJ, 628, 655

Vollmer, B. 2003, A\&A, 398, 525

Vollmer, B., Cayatte, V., Boselli, A., Balkowski, C., \& Duschl, W. J. 1999, A\&A, 349,411

Vollmer, B., Cayatte, V., Balkowski, C., \& Duschl, W. J. 2001, ApJ, 561, 708

Vollmer, B., Balkowski, C., Cayatte, V., van Driel, W., \& Huchtmeier, W. 2004a, A\&A, 419, 35

Vollmer, B., Beck, R., Kenney, J. D. P., \& van Gorkom, J. H. 2004b, AJ, 127, 3375

Werner, N., de Plaa, J., Kaastra, J. S., et al. 2006, A\&A, 449, 475 